\title{
A convenient synthetic approach to 5-(het)arylhydrazine substituted 1,2,4-triazines
}

\author{
A. P. Krinochkin ${ }^{\text {ab }}$, M. R. Guda ${ }^{a}$, \\ A. Rammohan ${ }^{\mathrm{a}}$, D.S. Kopchuk ${ }^{\mathrm{ab}}$, \\ G. V. Zyryanov ${ }^{a b^{*}}$, V.L. Rusinov ${ }^{a b}$, O. N. Chupakhin ${ }^{a b}$ \\ ${ }^{a}$ Ural Federal University named after the first President of Russia B. N. Yeltsin, \\ 19 Mira st., Ekaterinburg, 620002, Russian Federation \\ ${ }^{b} I . Y a$. Postovsky Institute of Organic Synthesis of RAS (Ural Branch), \\ 22/20 S. Kovalevskoy/Akademicheskaya st., Ekaterinburg, \\ 620990, Russian Federation \\ *email: g.v.zyrianov@urfu.ru
}

Abstract. A convenient synthesis of 1,2,4-triazines bearing the moieties of (hetero) arylhydrazines at the position of $\mathrm{C} 5$ of the 1,2,4-triazine core is reported.

Keywords: 1,2,4-triazines; (hetero)arylhydrazines; 5-cyano-1,2,4-triazines; ipso-substitution Received: 09.09.2020. Accepted: 20.12.2020. Published:30.12.2020.

(c) Krinochkin A. P., Guda M. R., A. Rammohan, Kopchuk D. S., Zyryanov G. V., Rusinov V. L., Chupakhin 0. N., 2020

\section{Introduction}

1,2,4-Triazines with moieties of (hetero)arylhydrazines at C5 position are of interest as substrates for further heterocyclizations for the synthesis of different condensed triazines, e.g., $2 \mathrm{H}$ pyrazolo $[3,4-e][1,2,4]$ triazin- $7(6 H)-$ ones, 2,6-dihydro-[1,2,4] triazino[5,6- $d]$ $[1,2,3]$ triazin-4-amines [1] or 2,6-dihydro-[1,2,4] triazino[6,5-e][1,2,4]triazines [2]. As for the methods for the synthesis of similar compounds it was previously proposed ipso-substitution of (methyl) sulfanyl group by arylhydrazine [2]. Also, there is a number of heterocyclizations of 1,2,4-triazine ring with the desired substituent at $\mathrm{C} 5$ position $[1,3,4]$. In addition, the opening of the condensed furan ring at the positions of $\mathrm{C} 5$ and $\mathrm{C} 6$ of the triazine ring is possible under the action of arylhydrazine [5].
At the same time, a known approach to the preparation of various 1,2,4-triazines with the residues of various nucleophiles at position $\mathrm{C} 5$ is the ipsosubstitution of the C5-cyano group. This is due to the easy availability of 5-cyano1,2,4-triazines obtained via the nucleophilic substitution of hydrogen process [6]. Thus, the possibility of the substitution of the C5-cyano group by the residues of such nucleophiles as alcohols [7-9], amines [7,10,11], anilines [12-14], lithiumcarboranes [15], various $\mathrm{CH}$ acid residues $[16,17]$, hydrazides of carboxylic acids [18, 19] etc. was demonstrated earlier. In this article we wish to report the synthesis of 1,2,4-triaizines bearing (hetero)arylhydrazine moieties at position $\mathrm{C} 5$ by means of ipso-substitution of C5-cyanogroup in 1,2,4-triazines under the action of (het) arylhydrazines. 


\section{Experimental part}

NMR ${ }^{1} \mathrm{H}$ and ${ }^{19} \mathrm{~F}$ spectra were recorded on a Bruker Avance-400 spectrometer $(400 \mathrm{MHz})$, the internal standard was correspondingly $\mathrm{SiMe}_{4}$ or $\mathrm{CFCl}_{3}$. Mass-spectra (ionization type - electrospray) were recorded on a MicrOTOF-Q II instrument from Bruker Daltonics (Bremen, Germany). Elemental analysis was performed on a Perkin Elmer PE 2400 II CHN analyzer. The starting 5-cyano-1,2,4-triazines 1 were obtained according to the described method [8].

\section{A general procedure for the synthesis} of 5-(het)arylarylhydrazinyl substituted 1,2,4-triazines 2

The mixture of corresponding 5-cyano1,2,4-triazine $\mathbf{1}$ (1 $\mathrm{mmol})$ and the corresponding (het)arylhydrazine $(1.05 \mathrm{mmol})$ was stirred at $150{ }^{\circ} \mathrm{C}$ for $8 \mathrm{~h}$ in argon atmosphere. After cooling down the obtained residue was crashed and dissolved in chloroform (5-10mL). The final products were isolated by flash chromatography on $\mathrm{SiO}_{2}$ using chloroform as eluent.

2-(2-(3,6-Diphenyl-1,2,4-triazin5-yl)hydrazinyl)benzo[ $d]$ thiazole (2a). Yield $305 \mathrm{mg}$ (0.77 mmol, 77\%). NMR ${ }^{1} \mathrm{H}$ (DMSO- $d_{6}, \delta$, ppm): 2.42 (s, 3H, Me), $7.02-$ 7.09 and 7.22-7.28 (both $\mathrm{m}, 1 \mathrm{H}, \mathrm{H}-5,6$ (benzothiazole)), 7.43-7.47 (m, 1H, H-7 (benzothiazole)), 7.50-7.60 (m, 5H, Ph), 7.61-7.65 (m, 1H, Ph), 7.66-7.71 (m, 1H, $\mathrm{H}-4$ (benzothiazole)), 8.16-8.22 ( $\mathrm{m}, 2 \mathrm{H}$, $\mathrm{Ph}), 8.35-8.40$ (m, 2H, Ph), 10.84 (s, 1H,

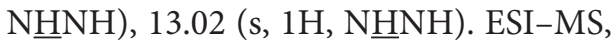
$\mathrm{m} / \mathrm{z}: 397.12(\mathrm{M}+\mathrm{H})^{+}$.

\section{Results and discussion}

The starting 5-cyano-1,2,4-triazines 1 were synthesized as described in literature [8]. Ipso-substitution reaction was carried out under the solvent-free condi-
2-(2-(3-(4-Fluorophenyl)-6-(p-tolyl)1,2,4-triazin-5-yl)hydrazinyl)benzo[d] thiazole (2b). Yield $310 \mathrm{mg}(0.72 \mathrm{mmol}$, 72\%). NMR ${ }^{1} \mathrm{H}$ (DMSO- $\left.d_{6}, \delta, \mathrm{ppm}\right): 2.42$ (s, 3H, Me), 7.07 and 7.28 (both ddd, ${ }^{3}$ J 7.6, $7.6 \mathrm{~Hz},{ }^{4} \mathrm{~J} 1.2 \mathrm{~Hz}, \mathrm{H}-5,6$ (benzothiazole)), 7.33-7.36 (m, 2H, $\left.\mathrm{CH}_{3} \mathrm{C}_{6} \underline{\mathrm{H}}_{4}\right), 7.40-7.45$ (m, $2 \mathrm{H}, \mathrm{C}_{6} \mathrm{H}_{4} \mathrm{~F}$ ), 7.45-7.48 (m, 1H, H-7 (benzothiazole)), 7.77-7.80 (m, 1H, H-4 (benzothiazole)), 8.03-8.06 (m, $2 \mathrm{H}, \mathrm{CH}_{3} \mathrm{C}_{6} \underline{\mathrm{H}}_{4}$ ), 8.49-8.54 (m, 2H, $\left.{ }_{6} \mathrm{H}_{4} \mathrm{~F}\right), 11.33$ (s, 1H,

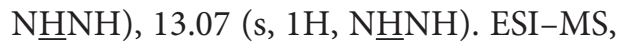
$\mathrm{m} / \mathrm{z}: 429.13(\mathrm{M}+\mathrm{H})^{+}$.

5-(2-(4-Fluorophenyl)hydrazinyl)3,6-di-p-tolyl-1,2,4-triazine (2c). Yield $310 \mathrm{mg}$ (0.80 mmol, 80\%). NMR ${ }^{1} \mathrm{H}$ $\left(\mathrm{CDCl}_{3}, \delta, \mathrm{ppm}\right): 2.43$ (s,3H, Me), 2.45 (s, 3H, Me), 6.90-6.96 (m, 2H, $\left.\mathrm{C}_{6} \mathrm{H}_{4} \mathrm{~F}\right)$, 6.98-7.02 (m, 2H, C $\left.{ }_{6} \mathrm{H}_{4} \mathrm{~F}\right), 7.25-7.28$ $\left(\mathrm{m}, 2 \mathrm{H}, \mathrm{CH}_{3} \mathrm{C}_{6} \mathrm{H}_{4}\right), 7.25-7.28(\mathrm{~m}, 2 \mathrm{H}$, $\left.\mathrm{CH}_{3} \mathrm{C}_{6} \underline{\mathrm{H}}_{4}\right), 7.29-7.33\left(\mathrm{~m}, 2 \mathrm{H}, \mathrm{CH}_{3} \mathrm{C}_{6} \underline{\mathrm{H}}_{4}\right)$, 7.75-7.78 (m, 2H, $\left.\mathrm{CH}_{3} \mathrm{C}_{6} \underline{\mathrm{H}}_{4}\right), 7.96-8.00$ (m, $\left.2 \mathrm{H}, \mathrm{CH}_{3} \mathrm{C}_{6} \underline{\mathrm{H}}_{4}\right), 8.79$ (s, $1 \mathrm{H}, \mathrm{N} \underline{\mathrm{HNH}}$ ), 9.22 (s, 1H, NHN $\underline{H})$. NMR ${ }^{19} \mathrm{~F}\left(\mathrm{CDCl}_{3}, \delta\right.$, ppm): -126.51 (s, 1F). ESI-MS, m/z: 386.18 $(\mathrm{M}+\mathrm{H})^{+}$.

5-(2-Phenylhydrazinyl)-3,6-di- $p$ tolyl-1,2,4-triazine (2d). Yield $280 \mathrm{mg}$ (0.76 mmol, 76\%). NMR ${ }^{1} \mathrm{H}$ (DMSO- $d_{6}$, $\delta, \mathrm{ppm}): 2.44$ (s, 3H, Me), 2.46 (s, 3H, Me), 6.61-6.67 (m, 1H, Ph), 7.03-7.08 (m, 2H, $\mathrm{Ph}), 7.10-7.16(\mathrm{~m}, 2 \mathrm{H}, \mathrm{Ph}), 7.24-7.30$ $\left(\mathrm{m}, 2 \mathrm{H}, \mathrm{CH}_{3} \mathrm{C}_{6} \mathrm{H}_{4}\right), 7.32-7.37(\mathrm{~m}, 2 \mathrm{H}$, $\left.\mathrm{CH}_{3} \mathrm{C}_{6} \underline{\mathrm{H}}_{4}\right), 8.00-8.05$ (m, $\left.2 \mathrm{H}, \mathrm{CH}_{3} \mathrm{C}_{6} \underline{\mathrm{H}}_{4}\right)$, 8.09-8.13 (m, 2H, $\left.\mathrm{CH}_{3} \mathrm{C}_{6} \underline{\mathrm{H}}_{4}\right), 9.10(\mathrm{~s}, 1 \mathrm{H}$,

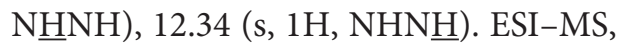
$\mathrm{m} / \mathrm{z}: 368.19(\mathrm{M}+\mathrm{H})^{+}$.

tions, as reported earlier for anilines and hydrazides [12, 18] (Scheme 1).

The structure of the obtained products 2 was confirmed by using NMR ${ }^{1} \mathrm{H}$, mass- 

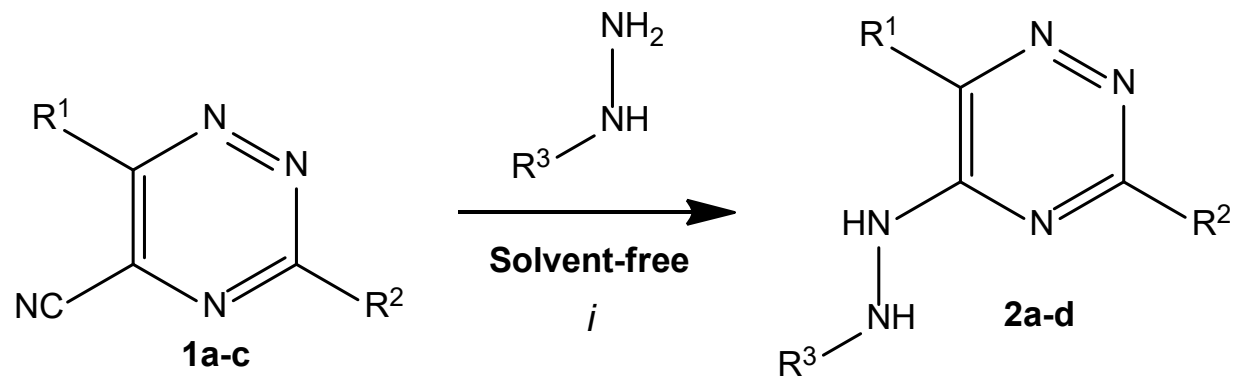

(a) $\mathrm{R}_{1}=\mathrm{R}_{2}=\mathrm{Ph} ; \mathrm{R}_{3}=$

(b) $\mathrm{R}_{1}=4$-Tol; $\mathrm{R}_{2}=4-\mathrm{FC}$

(c) $R_{1}=R_{2}=4-T o l ; R_{3}=4-F C$<smiles>Cc1nc2ccccc2s1</smiles>

i

(d) $\mathrm{R}_{1}=\mathrm{R}_{2}=4$-Tol; $\mathrm{R}_{3}=\mathrm{Ph}$<smiles>Cc1nc2ccccc2s1</smiles>

Scheme 1. Reagents and conditions: $150^{\circ} \mathrm{C}, 8 \mathrm{~h}$, argon atmosphere.

spectrometry and elemental analysis. Thus, NMR ${ }^{1} \mathrm{H}$ spectra of compound 2 contains two characteristic broadened singlets for $\mathrm{N}-\mathrm{H}$-protons in the area of 8.79-13.07 ppm, signals of protons of the substituents at the positions of $\mathrm{C} 3$ and $\mathrm{C} 6$ of the triazine moiety, as well as the signals of protons of (hetero)aromatic substituents of hydrazines.
It is worth to mention that, di(het)aryl hydrazine moieties are found in many natural and synthetic biologically active compounds [20-22]. Therefore, further studies are needed to evaluate the reactivity, biological activity and chelating properties of the obtained products 2 .

\section{Conclusions}

In conclusion, we have reported herein a convenient method for the synthesis of 3,6-disubstituted 1,2,4-triazines bearing moieties (het)arylhydrazine residues at the position of $\mathrm{C} 5$ of the triazine core

by means of ipso-substitution of cyanogroup in 1,2,4-triazine-5-carbonitriles by (het)arylhydrazines under the solvent-free conditions.

\section{References}

1. Al-Matar HM, Khalil KD, Al-Dorri DM, Elnagdi MH. Efficient routes to pyrazolo[3,4-e][1,2,4]triazines and a new ring system: $[1,2,4]$ triazino[5,6- $d]$ [1,2,3]triazines. Molecules. 2010;15:3302-10. doi:10.3390/molecules 15053302

2. Heunhoeffer H, Reichel D. Triazino[6,5-e[-1,2,4-triazine; II. Synthesis. 1988;11:877-9. doi:10.1055/s-1988-27734 
3. Molina P, Tárraga A, Espinosa A, Lidón M. J. Oxamic Acid Derivatives in Heterocyclic Synthesis: Preparation of 1,4-Oxazine and 1,2,4-Triazino[5,6-e[[1,3,4] oxadiazine Derivatives. Synthesis. 1987;2:128-34.

doi:10.1055/s-1987-27860

4. Sayed AR. Synthesis of 1,3,4-thiadiazines, bis-1,3,4-thiadiazoles, [1,2,4] triazino[3,4-b] $[1,3,4]$ thiadiazine, thiazolines from carbonothioic dihydrazide. Tetrahedron. 2012;68:2784-9.

doi:10.1016/j.tet.2012.02.011

5. Juhász-Riedl Zs, Hajós Gy, Kollenz G, Messmer A. Synthesis and ring transformation of a new fused as triazinium salt. Chem Ber. 1989;122:1935-8.

doi:10.1002/cber.19891221018

6. (a) Charushin VN, Chupakhin ON. Nucleophilic C-H functionalization of arenes: a contribution to green chemistry. Russ Chem Bull Int Ed. 2019;68:453-71.

doi:10.1007/s11172-019-2441-3; (b) Chupakhin ON, Rusinov VL, Ulomsky EN, Kojevnikov DN, Neunhoeffer H. Nucleophilic substitution of hydrogen in the reaction of 1,2,4-triazine-4-oxides with cyanides. Mendeleev Commun. 1997;7:66-7. doi:10.1070/MC1997v007n02ABEH000700

7. Huang JJ. Synthesis of Fused 1,2,4-Triazines: 6-and 7-Azapteridine and 6-Azapurine Ring Systems. J Org Chem. 1985;50:2293-8.

doi:10.1021/jo00213a019

8. Kozhevnikov DN, Kozhevnikov VN, Kovalev IS, Rusinov VL, Chupakhin ON, Aleksandrov GG. Transformations of 1,2,4-Triazines in Reactions with Nucleophiles: V. SNH and ipso-Substitution in the Synthesis and Transformations of 5-Cyano1,2,4-triazines. Russ J Org Chem. 2002;38:744-50.

doi:10.1023/A:1019631610505

9. Savchuk MI, Khasanov AF, Kopchuk DS, Krinochkin AP, Nikonov IL, Starnovskaya ES, Shtaitz YK, Kovalev IS, Zyryanov GV, Chupakhin ON. New push-pull fluorophores on the basis of 6-alkoxy-2,2'-bipyridines: rational synthetic approach and photophysical properties. Chem Heterocycl Compds. 2019;55:554-9. doi:10.1007/s10593-019-02495-5

10. Rusinov VL, Zyryanov GV, Egorov IN, Ulomskii EN, Aleksandrov GG, Chupakhin ON. Synthesis of 8 -Aryl $[1,2,4]$ triazolo $[1,5-d][1,2,4]$ triazin- $5(6 H)$-ones by $\mathrm{S}_{\mathrm{N}}{ }^{\mathrm{H}}$ Reactions. Russ J Org Chem. 2004;40:85-9.

doi:10.1023/B:RUJO.0000034914.87626.86

11. Rykowski A, Branowska D, Makosza M, Van LyP. Reactions of 1,2,4-Triazines with Nitromethide Ion. A Convenient Method of Preparation of 1,2,4-Triazin5-ylcarbaldehyde Oximes and their Synthetic Applications. J Heterocycl Chem. 1996;33:1567-71.

doi:10.1002/jhet.5570330603

12. Kopchuk DS, Chepchugov NV, Kovalev IS, Santra S, Rahman M, Giri K, Zyryanov GV, Majee A, Charushin VN, Chupakhin ON. Solvent-free synthesis of 5-(aryl/alkyl) amino-1,2,4-triazines and $\alpha$-arylamino-2,2'-bipyridines with greener prospects. RSC Adv. 2017;7:9610-9.

doi:10.1039/C6RA26305D 
13. Kopchuk DS, Starnovskaya ES, Shtaitz YK, Khasanov AF, Kim GA, Nosova EV, Krinochkin AP, Zyryanov GV, Rusinov VL, Chupakhin ON. 5-Aryl-2,2'-bipyridines bearing fluorinated anilines residues at C6 position: synthesis and photophysical properties. Res Chem Intermed. 2020;48:3929-44.

doi:10.1007/s11164-020-04182-Z

14. Starnovskaya ES, Savchuk MI, Shtaitz YaK, Kopchuk DS, Kovalev IS, Taniya OS, Pavlyuk DE, Khasanov AF, Zyryanov GV, Chupakhin ON. Polynuclear Aromatic Amines as N-Nucleophiles in the ipso-Substitution of the Cyano Group in 1,2,4-Triazines. Russ J Org Chem. 2020;56:335-8.

doi:10.1134/S1070428020010268

15. Prokhorov AM, Kozhevnikov DN, Rusinov VL, Chupakhin ON, Glukhov IV, Antipin MY, Kazheva ON, Chekhlov AN, Dyachenko OA, Organometallics. 2006;25:2972-7. doi:10.1021/om051058v

16. Ohba S, Konno S, Yamanaka H. Studies on as-Triazine Derivatives: XVI: Reaction of 1,2,4-Triazinecarbonitriles with Carbanions. Chem Pharm Bull. 1991;39:486-8. doi:10.1248/cpb.39.486

17. Kozhevnikov DN, Kovalev IS, Prokhorov AM, Rusinov VL, Chupakhin ON. $\mathrm{S}_{\mathrm{N}}{ }^{\mathrm{H}}$ reactions of pyrazine $\mathrm{N}$-oxides and 1,2,4-triazine 4-oxides with $\mathrm{CH}$-active compounds. Russ Chem Bull Int Ed. 2003;52:1588-94. doi:10.1023/A:1025601311393

18. Kopchuk DS, Krinochkin AP, Starnovskaya ES, Shtaitz YaK, Kovalev IS, Zyryanov GV, Rusinov VL, Chupakhin ON. Substitution of Cyano Group in Position 5 of 1,2,4-Triazines by Carboxylic Acid Hydrazide Residues under Solvent-Free Conditions. Russ J Org Chem. 2018;54:509-11. doi:10.1134/S1070428018030223

19. Krinochkin AP, Kopchuk DS, Giri K, Shtaitz YaK, Starnovskaya ES, Khalymbadzha IA, Drokin RA, Ulomsky EN, Santra S, Zyryanov GV, Rusinov VL, Chupakhin ON. A PASE Approach towards (Adamantyl-1)-, Alkyl- and (Het)Aryl-Substituted [1,2,4] triazolo[1,5-d][1,2,4]triazines: A Sequence of Two Solvent-Free Reactions Bearing Lower E-Factors. ChemistrySelect. 2018;3:8202-6.

doi:10.1002/slct.201801244

20. Rosen BR, Werner EW, O’Brien AG, Baran PS. Total Synthesis of Dixiamycin B by Electrochemical Oxidation. J Am. Chem Soc. 2014;136:5571-4. doi:10.1021/ja5013323

21. Zhang Q, Mandi A, Li S, Chen Y, Zhang W, Tian X, Zhang H, Li H, Zhang W, Zhang S, Ju J, Kurtan, T, Zhang C. N-N-Coupled Indolo-sesquiterpene Atropo-Diastereomers from a Marine-Derived Actinomycete. Eur J Org Chem. 2012;27:5256-62. doi:10.1002/ejoc.201200599

22. Klug DM, Diaz-Gonzalez R, Pe'rez-Moreno, G, Ceballos-Pe'rez G, Garc1'aHerna'ndez R, GomezPe'rez V. Evaluation of a class of isatinoids identified from a high-throughput screen of human kinase inhibitors as anti-Sleeping Sickness agents. PLoS Negl Trop Dis. 2019;13(2):e0007129.

doi:10.1371/journal.pntd.0007129 